\title{
Ulcus cruris oft von neuropathischem Schmerz begleitet
}

\author{
Unabhängig von der Ursache des Geschwürs leidet die Mehrzahl der \\ Patienten mit einem Ulcus cruris an neuropathischen Schmerzen.
}

chmerzen sind eine häufige Begleiterscheinung von Beingeschwüren. Und glaubt man den Ergebnissen einer französischen Studie, ist die oft vertretene Ansicht, venöse Beinulzera seien selten schmerzhaft, nicht länger haltbar. $56 \%$ der befragten Patienten mit Ulcus cruris venosum, die für die Studie untersucht wurden, hatten nachweislich neuropathische Schmerzen, also eine Punktzahl von mindestens 4 (von maximal 10) im DN4-Diagnosefragebogen zum neuropathischen Schmerz.

Bei Patienten mit Ulcus cruris arteriosum lag die Quote für neuropathische Schmerzen sogar bei $75 \%$. Für gemischte Ulzera gaben die Forscher den Anteil mit $64 \%$ an. Im Mittel lag die Rate bei
$58 \%$. Die Ursachen, die den offenen Beinen zugrunde lagen, hatten keinen Einfluss auf die DN4-Werte. Aus den erhobenen Daten ließ sich auch nicht ableiten, welche Patienten mit Ulcus cruris neuropathische Schmerzen entwickeln werden und welche nicht.

Fast jeder dritte der 81 Studienpatienten gab die Schmerzstärke auf einer bis 10 ansteigenden visuellen Analogskala mit mehr als 5 und damit als mindestens mittelstark, jeder fünfte mit mehr als 7 und somit als stark an. Drei Viertel der Patienten wurden gegen ihre Schmerzen behandelt, $30 \%$ bekamen Opioide. Aber nur wenige erhielten Mittel wie Pregabalin oder Gabapentin, die spezifisch gegen neuropathische Schmerzen wirken.
Fazit: Neuropathische Schmerzen bei venösen Beinulzera sind häufig. Die Resultate sollten Ärzte ermutigen, Patienten mit Beinulzera regelhaft auf neuropathische Schmerzen zu untersuchen, so die Autoren. Helfen kann dabei der schon erwähnte DN4-Fragebogen. Er enthält Fragen nach dem Schmerzcharakter (Brennen? Kälteschmerz? Elektrisierende Schläge?) und Begleitsymptomen (Kribbeln? Ameisenlaufen? Taubheit? Jucken?). Danach wird eingetragen, ob eine Hypästhesie auf Berührung und/oder Stiche in der schmerzenden Region besteht und ob der Schmerz durch Bestreichen etwa mit einem Pinsel ausgelöst oder verstärkt wird. Ab einem Wert von 4 Punkten ist von einem neuropathischen Schmerz auszugehen, die Sensitivität liegt für diesen Schwellenwert bei $83 \%$ und die Spezifität bei $90 \%$.

Dr. Robert Bublak

Eusen M et al. Neuropathic pain in patients with chronic leg ulcers. J Eur Acad Dermatol Venereol 2016; 30: 1603-5

\section{Neue Therapieoption bei atopischer Dermatitis}

\section{Die topische Behandlung von atopischer Dermatitis basiert seit Jahren auf Kortikosteroiden, Calcineurin-Inhibitoren oder systemischen Immunsuppressiva. Der Januskinase-Inhibitor Tofacitinib könnte laut einer kanadischen Studie eine neue Behandlungsoption sein.}

$\mathrm{D}$ ie Januskinase (JAK) spielt eine entscheidende Rolle bei der Übermittlung von Signalen, die die inflammatorische Antwort bei Patienten mit atopischer Dermatitis (AD) befeuern. Tofacitinib hemmt die JAK, was wiederum die Freisetzung von Interleukin (IL)-4 und IL-31 blockiert, die unter anderem für den Pruritus verantwortlich sind. Kanadische Forscher haben nun die erste randomisierte klinische Studie mit dem topischen niedermolekularen JAK-Inhibitor durchgeführt.

An der doppelblinden Phase-IIa-Studie nahmen 69 Erwachsene teil, die unter milder bis moderater AD (PGA = Physician's Global Assessment, 2-3) litten. Bei allen Teilnehmern lag seit über sechs Monaten die Diagnose einer klinisch stabilen $\mathrm{AD}$ vor. Im Mittel waren die Patienten 31,4 Jahre alt und die AD bedeckte etwa 6,8\% ihrer Körperoberfläche (BSA = „body surface area“).

Die Teilnehmer waren dazu angehalten, die betroffenen Hautstellen zweimal täglich mit $2 \%(20 \mathrm{mg} / \mathrm{g})$ TofacitinibSalbe oder einer Salbe ohne Wirkstoff einzucremen. Nach vier Wochen topischer Behandlung reduzierte sich der EASI(Eczema Area and Severity Index) -Wert unter Tofacitinib signifikant stärker als mit der Salbengrundlage allein (-81,7\% vs. $-29,9 \%)$. Auch der Anteil der Patienten, die nach der vierwöchigen Behandlung eine erscheinungsfreie oder nahezu erscheinungsfreie Haut aufwiesen, war mit Tofacitinib signifikant höher (73\% vs. $22 \%)$. Dementsprechend sank auch der BSA-Wert bei den Tofacitinib-Behandelten deutlich um 76\% (Kontroll-Gruppe $31 \%$ ). Sowohl die BSA als auch die PGA-Werte der Patienten waren unter dem JAK-Inhibitor bereits ab der ersten Anwendungswoche signifikant reduziert.

Die häufigsten Nebenwirkungen waren Nasopharyngitis, Kopfschmerz und Juckreiz oder Schmerzen an der Applikationsstelle, die sowohl in der Verum- als auch in der Gruppe mit wirkstofffreier Salbe auftraten. Schwere oder gefährliche $\mathrm{Ne}$ benwirkungen wurden nicht beobachtet.

Allerdings handelte es sich um eine Kurzzeitstudie mit einer relativ geringen Teilnehmerzahl. Breiter angelegte weiterführende Studien könnten helfen, den JAK-Inhibitor als neue Therapieoption voranzutreiben.

Fazit: Die Hemmung der Januskinase scheint ein vielversprechendes therapeutisches Target in der Therapie der AD zu sein. Die topische Behandlung mit Tofacitinib über vier Wochen verbesserte den EASI-Score signifikant, wirke rasch und hatte ein akzeptables Sicherheitsprofil.

Julia Rustemeier

Bissonnette $\mathrm{R}$ et al. Topical tofacitinib for atopic dermatitis: a phase lla randomized trial. $\mathrm{Br} \mathrm{J}$ Dermatol 2016; 175: 902-11 